Results Data were collected for 51 patients (63\% female; mean age at surgery 14.7 years). Diagnoses included adolescent $(62.8 \%)$, juvenile $(7.8 \%)$ and infantile (5.9\%) idiopathic scoliosis, congenital (7.8\%) and syndromic scoliosis (2\%) and Scheuermann's kyphosis (13.7\%). Mean $( \pm S D)$ thoracic curvature was $61.3 \pm 15.2^{\circ}$, and mean kyphosis in those with Scheuermann's was $92.4 \pm 7.3^{\circ}$. No correlation was elicited between FEV1 or FVC (\%predicted) and VO2peak(\%predicted). Greater thoracic curves were associated with lower FEV1(\%predicted), $\quad \mathrm{r}=-0.45, \quad \mathrm{p}=0.02, \quad$ smaller total lung capacity $(r=-0.47, p=0.002)$ and lower $B R$ at the end of exercise $(\mathrm{r}=-0.57, \mathrm{p}<0.0001)$. There was a positive correlation between FEV1(\%predicted) and BR $(r=0.65, p<0.001)$ and a negative correlation between FEV1 and VO2 at AT $(\mathrm{r}=-0.37, \mathrm{p}=0.008)$. Those with higher VO2peak measures also had higher VO2 at AT $(r=0.78, p \leq 0.001)$. SRS-22 scores correlated significantly with VO2peak(\%predicted) (total SRS22 vs VO2peak(\%predicted), $\mathrm{r}=0.50, \mathrm{p}=0.006$ ).

Conclusion Contrary to previously published data, those with larger thoracic curves and therefore poorer lung function show improved exercise capacity and later onset AT, likely as a consequence of improved physical conditioning. These patients also report better quality of life, which may be due to maintenance of normal exercise levels. These findings suggest that physical adaptation occurs in scoliosis with impaired lung function, and that this may be protective to patients' mental health.

\section{G446 HIGHLY ELEVATED FERRITIN LEVELS ARE ASSOCIATED WITH HAEMOPHAGOCYTIC LYMPHOHISTIOCYTOSIS - ARE WE MISSING TREATABLE DIAGNOSES? A RETROSPECTIVE SERVICE EVALUATION OF DIAGNOSIS IN PATIENTS WITH FERRITIN $>10,000 \mu \mathrm{G} / \mathrm{L}$}

${ }^{1} \mathrm{E}$ Sen, ${ }^{2} \mathrm{~L}$ Moran, ${ }^{3} \mathrm{~B}$ Almeida, ${ }^{4} \mathrm{R}$ Close, ${ }^{5} \mathrm{~J}$ Bennett, ${ }^{6} \mathrm{C}$ Anderson, ${ }^{7} \mathrm{~S}$ Deepak, ${ }^{8} \mathrm{R}$ Tattersall. ${ }^{1}$ Department of Paediatric Rheumatology, Bristol Royal Hospital for Children, Bristol, UK ${ }^{2}$ Department of Paediatric Rheumatology, Alder Hey Children's Hospital, Liverpool, UK; ${ }^{3}$ Department of Paediatric Rheumatology, Great Ormond Street Hospital for Children NHS Trust, London, UK; ${ }^{4}$ Department of Paediatric Rheumatology, Norfolk and Norwich University Hospital, Norwich, UK; ${ }^{5}$ Department of Paediatric Rheumatology, Great North Children's Hospital, Newcastle upon Tyne, UK; ${ }^{6}$ Department of Paediatric Rheumatology, Royal Hospital for Children, Glasgow, UK; ' Department of Paediatric Rheumatology, Nottingham Children's Hospital, Nottingham, UK; ${ }^{8}$ Department of Adolescent Rheumatology, Sheffield Children's Hospital, Sheffield, UK

\subsection{6/archdischild-2018-rcpch.435}

Background Haemophagocytic lymphohistiocytosis (HLH) is a hyperinflammatory syndrome which can complicate sepsis, malignancy or autoimmune disease and may lead rapidly to critical illness and death. Early treatment reduces mortality but diagnosis requires a high index of suspicion and correct interpretation of laboratory results. Highly elevated ferritin levels (HEF) $>10,000 \mu \mathrm{g} / \mathrm{L}$ are highly specific for HLH and should prompt consideration/exclusion of hyperinflammation. Diagnostic guidelines for HLH were published in 2004 requiring the presence of $\geq 5 / 8$ criteria.

Aim To assess recognition of HLH in a paediatric population with HEF.

Methods This retrospective study was conducted at 8 UK centres. Biochemistry databases identified patients $\leq 16$ years with serum ferritin $>10,000 \mu \mathrm{g} / \mathrm{L}$ between 01.04 .2014 and 31.03.2017. A standardised proforma was used to collect data. Cases were assessed against the $2004 \mathrm{HLH}$ criteria. Due to limited access to some laboratory tests, modified criteria using a threshold of $\geq 4 / 5$ (excluding tissue haemophagocytosis, decreased natural killer cell function, increased soluble interleukin-2 receptor) were also applied.

Results Ninety-four patients $(60.6 \%$ male $)$ were identified. $36 \%$ were aged $<1$ year, 16\% 1-5 years, 34\% 6-12 years and $14 \%$ 13-16 years. Management was in neonatal/paediatric intensive care in $49.4 \%$. Diagnoses included: infections (29.8\%), malignancies (21.3\%), rheumatological (13.8\%), immunological (9.6\%) and cardiac surgery (6.4\%). A diagnosis of HLH was made by the treating clinical team in $30.9 \%$ and considered in the differential in a further $18.1 \%$. Just 5 patients had complete data available to score against all 8 2004 HLH criteria whereas 49 patients had data to score against the modified criteria. Using all available data:

- $14 / 94$ (14.9\%) met $\geq 5 / 8$ criteria and all these patients were diagnosed with HLH by the treating team.

- 33 (35.1\%) met $\geq 4 / 5$ criteria and $17(51.5 \%)$ of these were diagnosed with HLH by clinicians. HLH was not documented as being considered in the differential in 11 (33.3\%)

Overall mortality was $33.0 \%(31 / 94)$ but was $17.2 \%(5 / 29)$ in those patients diagnosed with HLH during their admission.

Conclusion Although HEF is highly specific for HLH, the diagnosis was made or considered in just half of paediatric patients with this laboratory result. Increased awareness of this potentially-lethal condition is likely to lead to earlier treatment and reduced mortality.

Acknowledgements The following authors also contributed to this work - EL Long and F McErlane, Department of Paediatric Rheumatology, Great North Children's Hospital, Newcastle upon Tyne, UK, K McLellan, Department of Paediatric Rheumatology, Royal Hospital for Children, Glasgow, UK, K Gallagher and P Bale, Department of Paediatric Rheumatology, Addenbrooke's Hospital, Cambridge, UK, K Mahmood and C Pain, Department of Paediatric Rheumatology, Alder Hey Children's Hospital, Liverpool, UK

\section{G447 THE CROSS-SECTIONAL AND LONGITUDINAL ASSESSMENT OF THE EFFECTS OF NURSERY ATTENDANCE AND BREASTFEEDING ON RESPIRATORY SYMPTOMS AND THEIR IMPACT ON QUALITY OF LIFE IN THE FIRST 22 MONTHS OF LIFE- RESULTS FROM THE LIVERPOOL BABY BREATHING STUDY}

J Stead, M Semple, B Griffith, R Pickles, S Patel, J Barclay. Women's and Children's Health Institute of Translational Medicine, University of Liverpool, Liverpool, UK

\subsection{6/archdischild-2018-rcpch.436}

Aims To determine the effects of breastfeeding and nursery attendance on respiratory symptoms and quality of life related to respiratory symptoms in infants from Liverpool over the first 22 months of life.

Methods The Liverpool Baby Breathing Study (LBBS) uses a parent completed respiratory symptom questionnaire, to assess respiratory symptoms in infants from four to twenty-two months of age. Infants were recruited from the Liverpool Women's Hospital between 23/01/2013 and 03/11/2014. Questionnaires were sent to participants at four, ten, sixteen and twenty-two months of age via post or an automated emailing system. The cross-sectional analysis of the LBBS was weighted to ensure the cohort's index of multiple deprivations was comparable to the eligible births. $\chi 2$, Mann-Whitney $U$ and 